\title{
STATE-OF-THE-ART AND TRENDS OF COMPUTER-AIDED INNOVATION TOOLS
}

Towards the integration within the Product Development Cycle

\section{Gaetano Cascini}

Dipartimento di Meccanica e Tecnologie Industriali, Università degli Studi di Firenze, via S. Marta, 3-50139 Florence, Italy

Abstract: The design of competitive products requires meeting several market demands often contradictory, or at least very hard to achieve due to the hard time constrains imposed by the competitors. For example, from a structural point of view, mechanical parts must be light-weighted as well as stiff and strong according to the application requirements. The integration of CAE tools is the basic step towards the fulfillment of these demands, but it must be observed that such integration involves just detailed design phases, while only stand alone tools are available to support the designer during the preliminary product development activities. In facts, nowadays, the market offers several tools to improve designer creativity and problem solving capabilities with a systematic approach. Nevertheless, it is worth to notice that current Computer-Aided Inventing (CAI) applications cannot be integrated with other product development systems. In this paper, a survey of CAI systems is presented with a set of hints about their future development towards the integration with Product Lifecycle Management (PLM) applications.

Key words: Computer-Aided Inventing, Systematic Innovation, Product Development Cycle, PLM

\section{INTRODUCTION}

The increasing demand for being competitive on the market has driven companies to drastically reduce product development cycles. At the same time, the growing of $\mathrm{CAD} / \mathrm{CAE}$ and virtual prototyping systems of the last decade has deeply modified the approach to design: the possibility to test varying technical solutions maintaining low costs and time has increased the 
level of confidence with which designers can propose "extreme" solutions. At the same time, the preliminary phases of product development cycle have been cut down in favor of testing solutions reached following a "trial and error" approach rather than adopting a systematic innovation process.

The combination of these issues has led to an unstable situation for companies that do not hold the monopoly in a specific industrial sector. The study presented by Miller and Morris ${ }^{1}$ shows that:

1. $10 \%$ only of North American companies has put on the market a new product in the last decade of XX century;

2. $90 \%$ of new products put on the market fail within four years from their appearance;

3. less than $1 \%$ of patents fully pay back the people who took on the investments;

4. $80 \%$ of successful innovation is proposed by customers instead of being developed by producers.

In order to improve the product development process and more specifically the innovation capabilities of a company, a systematic approach and suitable tools are needed also for the conceptual design phase. Nowadays, the market offers several tools to improve designer creativity and problem solving capabilities; among these, according to the author's experience, the most effective are methods and tools supporting the systematic transferring of innovative solutions among different technical areas by means of an abstraction of the process, i.e. the TRIZ theory and tools.

During the last years, major efforts have been dedicated to the integration of TRIZ with other methodologies. Among others: the criteria to adopt in order to combine TRIZ and Theory of Constraints benefits have been presented by several authors ${ }^{2,3}$. Innovative product development processes are being presented, which systematically integrate QFD with TRIZ and enable the effective and systematic creation of technical innovation for new products $^{4,5}$. It is known that the former is focused on the identification and the improvement of the most critical components of a mechanical system, and the latter is dedicated to the definition of a direct link between customer requirements and the most suitable inventive principles pointing at the solution of the corresponding technical problems. The synergy between QFD and TRIZ is extended also to Taguchi method with the goal of determining the design specifications for a product insensitive to uncontrolled influences ${ }^{6}$. Finally, still aiming at robust design practices, TRIZ and Axiomatic Design have been adopted in a pilot project by General Motors ${ }^{7}$ and the guidelines to combine TRIZ and Axiomatic Design in a Design for Six Sigma development process ${ }^{8}$. 
It is clear that all the above examples, as well as other published works, are focused on the integration of tools and methods for conceptual design. Besides, there's a lack of links with the product embodiment phases, even if some preliminary experiences have been approached ${ }^{9-11}$.

In this preliminary paper the main limits and opportunities for integrating existing Computer-Aided Inventing (CAI) tools with other Product Lifecycle Management (PLM) applications are surveyed; therefore the author's vision about the next generation of Product Development systems is presented, focusing on expected features and technology sore points. The full-length manuscript will be enriched with examples and explanations that are more detailed.

\section{PLM SYSTEMS BACKGROUND}

The evolution of Product Development tools has been characterized by different trends; the analysis of these trends offers useful hints for the prediction of next generation systems.

\subsection{Product Modeling Trend}

First, let's take into account CAD systems evolution (Fig. 1): the first generation was dedicated to explicit geometrical modeling with the transition from wireframe to canvas and solid modeling. These tools aimed at speeding-up technical representation tasks, but they didn't provide a useful support for designer, due to the big efforts required to revise geometry.

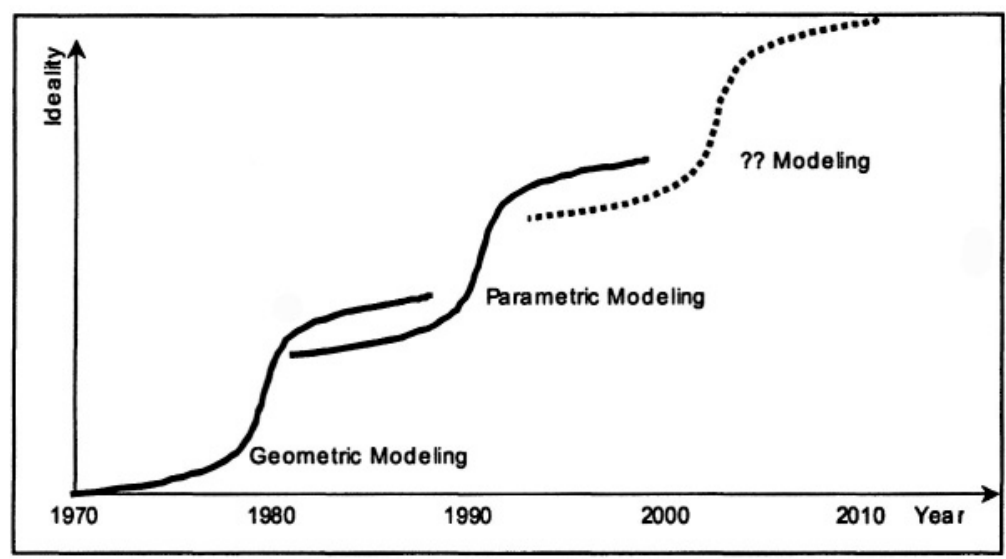

Figure 1. Product modeling evolution 
The step toward was given by CSG (Constructive Solid Geometry) representation, i.e. using solid primitives combined by means of Boolean operators. The model is stored in a tree with all the information about primitives and the way they are combined. The ability to edit the tree, i.e. the transition to parametric modeling, is a fundamental step to support the typical iterative process of design activity. The introduction of Boundary representation (B-rep), consisting in a description of solid geometry by means of its skin, mathematically expressed through NURBS, allowed the definition of complex shapes even with limited computation efforts.

From the user interface point of view, feature-based modeling changed the approach to CAD models definition, from geometry to technologycentric: geometric entities are now grouped according to the technological meaning of the shape element.

\section{$2.2 \quad$ Task-to-Process trend}

A second relevant trend is the transition from task-oriented applications to process-oriented systems: the former CAE tools were able to speed-up and sometimes automate several engineering tasks, but the integration was limited to product data exchange formats. Such a heterogeneous and fragmented system led to the introduction of Product Data Management (PDM) systems, i.e. tools for the management of any kind of product related information and their corresponding workflow. The main limit is here represented by the poor integration with Computer-Aided-tools apart from CAD systems.

As a consequence, PLM systems have emerged as a "strategic business approach that applies a consistent set of business solutions in support of the collaborative creation, management, dissemination, and use of product definition information across the extended enterprise from concept to end of life - integrating people, processes, business systems and information"12.

It should be observed that actual PLM systems are effectively integrated just with CAD-CAE applications; therefore, their efficiency is still poor for the preliminary design phases. One of the purposes of the present work is evaluating the perspective of linking PLM with CAI systems, as depicted in Fig. 2.

\subsection{Knowledge integration trend}

A third pattern of evolution that can be observed is related to the TRIZ Law of Shortening of Energy Flow Path: technological systems evolve in the direction of shortening the energy passage through the system. 
A complete system is usually constituted by four components: Working Tool, Transmission, Energy Source, and Control. During the first stages of CAD evolution, major efforts have been dedicated to the Working Tool, i.e. the geometric kernel; then, the information flow (Transmission) has been improved in terms of Modeling Features capabilities, making CAD systems closer to the "Energy Source" (Knowledge).

Even the evolution of Engineering Knowledge Management (EKM) systems can be attested at the third generation ${ }^{13}$ : starting from Content Management tools, whose intent was to support structured information management without any direct connection to product data, the following generation was focused on Design Automation, by means of tools capable of automating specific design tasks. Actually the third generation is at its infancy stage: EKM systems play the role of knowledge based automation systems capable of guiding different kind of product development tools (i.e.: CAD, FEM, PDM etc.) to create ad hoc automatic applications. Such an objective is approached by storing parameters and rules in relational databases and capturing others' systems functions directly. Once more the integration of EKM systems with Conceptual Design and Systematic Innovation tools is quite poor and further developments are needed (Fig. 2).

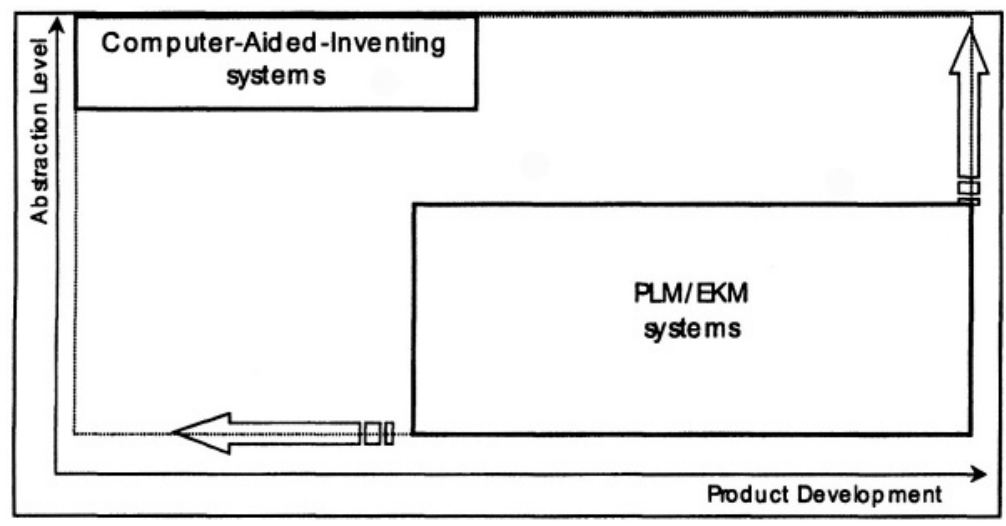

Figure 2. Toward the integration of product development systems

\section{CAI-PLM SYSTEMS EXPECTED EVOLUTION}

The goal of integrating CAI and PLM systems requires the development of a common platform for product data exchange: in other words all these tools must share the same product model. 
A first attempt toward the integration fulfillment has just been approached by the author together with several Italian universities through the research project "From systematic innovation to integrated product development". The integration objective of the above-mentioned tools is based on the introduction of Topological Optimization systems as a bridge capable of generating optimal geometrical solutions.

Therefore, the project concerns two main tasks, as depicted in Fig. 3:

1. systematize the translation of the functional model of a system and of its design requirements into an optimization problem; that means identifying design variables, defining an objective function, defining design constraints;

2. Define a Best Practice for the integrated use of topological optimization tools together with current PLM systems; that implies the definition of procedures for translating the topological optimization results into a geometry defined by "technological" features.

Since optimization techniques look for the "optimal" solution to a suitably coded problem, a critical aspect of the research is represented by the rigorous definition of the system to be optimized: if such a task is not properly accomplished the achievement of satisfactory results can be definitely compromised. The problem formulation of an optimization task is actually demanded to designer experience and very often, the underlying criteria are not elicited. Therefore, the main purpose of the author work is defining a set of criteria to formulate with a systematic and rigorous approach an optimization problem. In other words, it is necessary to define how to translate the functional architecture of a machine and its requirements into an optimization problem, i.e. identifying design variables and defining an objective function and design constraints.

A useful contribution is provided by the techniques for establishing which components or subassemblies of a system are more critical from functional, safety, reliability, and cost viewpoints where Axiomatic Design and FMEA analyses are combined in order to identify the components requiring an optimization process ${ }^{14}$.

With this perspective, axiomatic design tools efficiently aid the designer in capturing, analyzing, and decomposing requirements to be adopted for the optimization problem formulation. Moreover, the adoption of TRIZ based tools leads the designer to the definition of an ideal system architecture and consequently to the formulation of the objectives for each component and subassembly ${ }^{15}$.

The above presented objectives answer to requirements that are today not yet satisfied in product development processes, and they might bring advantages in terms of design time, cost and errors reduction, improvement in product quality, etc. Nevertheless, such an approach will not provide a full 
integration between CAI and PLM systems, since its top-down approach does not admit the use of CAI tools in specific design tasks with a direct link.

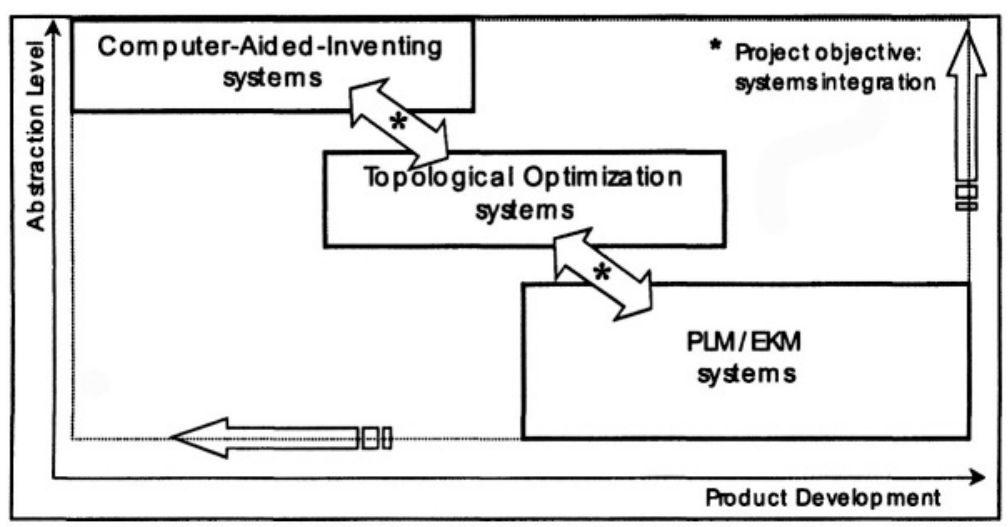

Figure 3. A proposal for the integration of product development systems

On the basis of the trends followed by Product Development tools described in the previous section, it is possible to anticipate the next generation of integrated engineering systems, according to the patterns described below.

The product modeling approach (Fig. 1) must shift to a higher abstraction level, in order to establish a direct link between product data at the conceptual design stage and detailed design; the most suitable is functional modeling for several matters:

- several CAI tools have already adopted such a technique for product modeling; hopefully they will enrich their capabilities in order to manage more complex hierarchies of functional models as well as relationships among the functions, i.e. decomposed-into, conditioned-by, enhanced-by and described-as relations ${ }^{16}$;

- functional modeling is history independent, which means that design elements added, subtracted, and modified in any sequence will always generate the same product model, therefore effectively providing useful means for simultaneous engineering, as already implemented by ImpactXoft ${ }^{17}$;

- functional modeling is much more powerful in capturing designer's intent, therefore codifying his implicit knowledge;

- the explicit association of geometrical features and functions allows automating the abstraction process from a specific technical system to a generic model of the problem to be solved, therefore ensuring a bidirectional integration between CAI and other PLM systems. 
Further advantages deriving from the adoption of functional modeling as the product representation technique are related to the knowledge integration trend presented in section 2. In facts, semantic processing technology is already used for enriching the knowledge base of CAI systems, by extracting from technical documents the solutions capable to accomplish a given function $^{18}$. Moreover, the semantic analysis of technical documents and patents can be pushed to the automatic extraction of functional models of a technical system ${ }^{19,20}$.

According to the aforementioned knowledge integration trend, such a path can lead to the full "encapsulation" of the Energy Source (Knowledge) into the PLM environment. The ideal final result of such a trend is a selfoperating design system, while the user should perform just "control" tasks, by selecting and not defining the most suitable solution.

It is worth to notice that in the recent past several approaches have been proposed for building Intelligent CAD systems; nevertheless, they are affected by severe limitation, mainly due to the formalism rigidity of symbolic approaches and the quest for full design automation rather than a realistic active support to the design process ${ }^{21}$.

Therefore, it is useful to distinguish between routine and inventive design tasks, the former class constituted by any engineering activity with all the parameters and variables known a priori or related by strictly defined rules.

While the automation of routine design tasks is already accomplished by the state of the art EKM technologies, trying to automate inventive tasks is a wrong objective; in other words, software systems can help inventing, but not invent! The characteristic of a design thought process to be something vague, fluid, amorphous cannot be constrained by rigid formalisms.

According to this statement, a conflicting requirement is requested to the next generation of CAI systems: they must embody a formalized Knowledge Base in order to suggest a set of solutions to the designer, but they must leave the maximum freedom to his way of thinking, even if pointing to a reliable direction, as operated by standard TRIZ tools.

This means enlarging the domain of routine design tasks, by linking functional requirements with sets of geometric features capable of maintaining the consistency of their functionality when assembled in a specific embodiment. This goal can be supported by the emerging technology for managing digital CAD libraries ${ }^{22}$ and 3D shapes searches ${ }^{23}$, so that discarding non-matching geometries can reduce the number of candidate shapes for accomplishing a given function.

On the other side, when approaching a real inventive problem, it is necessary to leave to the designer as much freedom as he was working with a pencil and a blank sheet. During the design process, a person needs to create a visual representation, even for abstract and verbal ideas, and then 
respond to it perceptually to discover new arrangements and shapes representing new ideas ${ }^{9}$. This requirement is still more urgent for team working. In a previous paper ${ }^{11}$, the author suggested the introduction of "CAD storming" practices: work together on a same geometrical model with the ability of applying deep changes to the geometry in absolute freedom. Such a cooperative work is actually limited by the slowness of modeling activity that counteracts brainstorming agility. Since changing easily and quickly, the model geometry is still not possible a "partial action" is proposed: by means of a common speech recognition module, the comments of the design team could be automatically translated into $3 \mathrm{D}$ primitive shapes to store in a more effective way the proposed solutions to be evaluated. Such a practice could be used also to track the thought process and effectively capture the design intent by linking verbal expressions with the developed geometry.

\section{CONCLUSIONS}

The role of CAI systems in the Product Development process will rapidly grow since product innovation has become the focus of any company's strategy.

Nevertheless state of the art CAI systems are still structured as standalone tools, while the need for reaching the objective in the shortest possible time (time-to-market) and with the maximum user perceived value (time to value) requires the full integration of Product Development systems.

In this paper, a survey of PLM tools evolution is presented and the opportunities to integrate CAI and PLM systems are evaluated with some hints about directions for their development.

An extended version of the paper will be presented at the IFIP World Computer Congress 2004, with further details and some examples about the proposed concepts.

\section{REFERENCES}

1. Miller, W. L., Morris, L., 4th Generation R\&D : Managing Knowledge, Technology, and Innovation (John Wiley \& Sons, 1999).

2. Stratton, R., Warburton, R.D.H., The strategic integration of agile and lean supply, International Journal of Production Economics, v85, n2, 183-198 (2003).

3. Rantanen, K., Domb, E., Simplified TRIZ - New Problem-Solving Applications for Engineers and Manufacturing Professionals (St. Lucie Press, 2002).

4. Yamashina, H., Ito, T., Kawada H., Innovative product development process by integrating QFD and TRIZ”, International Journal of Production Research, v40, n5,10311050 (2002). 
5. Tan, R., Kraft, D., A conceptual design methodology for variety using TRIZ and QFD, Proceedings of the ASMS 14th International Conference on Design Theory and Methodology Integrated Systems Design, , Sep 29-Oct 2 2002, Montreal, Canada.

6. Terninko, J., The QFD, TRIZ and Taguchi Connection: Customer-Driven Robust Innovation, Transactions of Ninth Symposium on Quality Function Deployment, Novi, MI QFD Institute (1997).

7. Hu, M., Yang, K., Taguchi, S., Enhancing Robust Design with the Aid of TRIZ and Axiomatic Design, Part I and Part II, The TRIZ Journal (October-November 2000).

8. Kai Y., Basem S. E. , Design for Six Sigma: A Roadmap for Product Development (McGraw-Hill Professional, 2003).

9. Leon, N., A proposal to integrate TRIZ and CAD (Computer Aided TRIZ-based Design), Proceedings of TRIZCON 2001.

10.Cascini, G., Pieroni, N., Rissone, P., Plastics design: integrating TRIZ creativity and semantic knowledge portals, Journal of Engineering Design, v15, n3, (2004).

11. Abate, V., Cascini, G., CAD for Conceptual Design: Forecasted Evolution, The TRIZ Journal (May 2003); http://triz-journal.com.

12.CIMdata, PLM Market Analysis Report (2002); http://www.cimdata.com/index.htm.

13.Pulli, M., Engineering Knowledge Management Systems evolution, PhD Thesis, University of Parma (2004).

14.Arcidiacono, G., Delogu, M., The integration of reliability techniques as an aid to the design optimisation of an earth moving machine, Proceedings of the 2001 ASMS International Mechanical Engineering Congress and Exposition, v 112, 157-164 (2001).

15. Cavallucci, D., Lutz, P., Kucharavy, D., Converging in problem formulation: A different path in design, Proceedings of the $14^{\text {th }}$ ASME International Conference on Design Theory and Methodology Integrated Systems Design, Montreal, Canada, Sep 29-Oct 2 (2002).

16.Shimomura, Y., Yoshioka, M., Takeda, H., Umeda, Y., Tomiyama, T., Representation of Design Object Based on the Functional Evolution Process Mode, Journal of Mechanical Design (ASME), Vol. 120 (June 1998).

17.Krouse, J., Simultaneous Product Development, ImpactXoft White Paper (2002); http://www.impactxoft.com.

18. Verbitsky, M., Semantic TRIZ, The TRIZ Journal (February 2004); http://triz-journal.com.

19. Cascini, G., Rissone, P., PAT-Analyzer: a tool to speed-up patent analyses with a TRIZ perspective, Proceedings of the ETRIA World Conference: TRIZ Future 2003, Aachen, Germany, November 12-14 (2003).

20.Cascini, G., Fantechi, A., Spinicci, E., Natural Language Processing of Patents and Technical Documentation, submitted for publication to the IAPR Workshop on Document Analysis Systems - University of Florence, Italy, September 8-10 (2004).

21.Bento, J., Feijó, B., An-agent based paradigm for building intelligent CAD systems, Artificial Intelligence in Engineering, v 11, 231 -244 (1997).

22.Reglil, W.C., Cicirello, V.A., Managing digital libraries for computer-aided design, Computer-Aided Design, v32,119-132 (2000).

23.Iyer, N., Kalyanaraman, Y., Lou, K., Jayanti, S., Ramani, K., A reconfigurable 3D engineering shape search system - Part I: shape representation, Proceedings of DETC' 03 ASME 2003 Design Engineering Technical Conferences and Computers and Information in Engineering Conference, Chicago, Illinois, USA, September 2-6 (2003). 